\title{
Value of supplier's capacity information in a two-echelon supply chain
}

\author{
İsmail Serdar Bakal • Nesim Erkip • Refik Güllü
}

Published online: 11 August 2011

(C) Springer Science+Business Media, LLC 2011

\begin{abstract}
In traditional supply chain models it is generally assumed that full information is available to all parties involved. Although this seems reasonable, there are cases where chain members are independent agents and possess different levels of information. In this study, we analyze a two-echelon, single supplier-multiple retailers supply chain in a single-period setting where the capacity of the supplier is limited. Embedding the lack of information about the capacity of the supplier in the model, we aim to analyze the reaction of the retailers, compare it with the full-information case, and assess the value of information and the effects of information asymmetry using game theoretic analysis. In our numerical studies, we conclude that the value of information is highly dependent on the capacity conditions and estimates of the retailers, and having information is not necessarily beneficial to the retailers.
\end{abstract}

Keywords Value of information · Limited capacity · Rationing game

\section{Introduction}

Consider a set of retailers with a common supplier. The retailers are independent agents serving geographically distant markets. The common supplier (from hereon 'supplier') has a restricted capacity to serve the set of retailers in question. In many industries, it is infeasible for the supplier to build capacity that is sufficient to cover all possible demands of the

\footnotetext{
İ.S. Bakal $(\bowtie)$

Department of Industrial Engineering, Middle East Technical University, 06531, Ankara, Turkey

e-mail: bakal@ie.metu.edu.tr

N. Erkip

Department of Industrial Engineering, Bilkent University, 06800, Ankara, Turkey

e-mail: nesim@bilkent.edu.tr

R. Güllü

Industrial Engineering Department, Bogaziçi University, 34342, Istanbul, Turkey

e-mail: refik.gullu@boun.edu.tr
} 
downstream members of the supply chain, especially when demand is highly uncertain and capacity expansion is costly or infeasible in the short or medium term. Hence, there is always a possibility that orders will exceed capacity. In such cases, the supplier cannot satisfy the orders in full and should somehow allocate its capacity among them.

In this context, we consider a supply chain where the supplier has a pre-announced allocation policy to ration the supplied product among the retailers, in case the total quantity requested by the retailers exceeds the supply. The objective is to find the ordering quantity for a retailer, and a non-cooperative game can be formed to determine the order quantities. This setting, where each retailer optimizes its own objective function, is known as 'the rationing game'. In the rationing game, it is usually assumed that all retailers know all pertinent information, including information about the supplier's capacity.

Specifically, we analyze a two-echelon supply chain with multiple retailers and a single supplier in a single-period model where the supplier has limited capacity to serve the retailers. There are independent, random demands observed at the retailers. At the beginning of the period, all retailers simultaneously place their orders and pay a down payment for the procurement cost for their entire orders. Following the result of Gurnani and Shi (2006) and without loss of generality, we say that the retailers pay for the entire procurement cost of their orders (we further discuss this issue at the end of Sect. 3, after we introduce the characterization of the expected costs of the retailers in (1) and (2)). The orders are not satisfied immediately as the manufacturer has a production and transportation lead time. After the lead time, the supplier allocates the quantity among the retailers and delivers their orders. If the orders cannot be delivered in full, the supplier refunds the procurement cost for the undelivered portion of the orders. It should be noted that the lead time we consider merely reflects the time between the advance payment and the capacity allocation. That is, the lead time is not long enough to cover more than one decision epoch or resolution of capacity uncertainty, but it is long enough so that tying up a large amount of cash may have financial consequences. In many cases, especially in supplier-driven supply chains, retailers are forced to make advance payments for their orders in order to secure some portion of the supplier's capacity. If the time between these advance payments and the receipt of goods is long, it places serious financial burdens on the retailers. We believe that our model description involving lead time addresses this issue.

Using down payments is common in practice and well explored in the literature. Kiyotaki and Moore (1997) describe the economic rationale behind using down payments in creditrequiring operations (see pages 10 and 11 of the mimeo). The down payment considered is in terms of a fixed proportion of the total procurement cost. Cvsa and Gilbert (2002) mention the idea of franchise fees, which is a constant (not a percentage of the total procurement value). In both cases, this prepayment, regardless of the amount paid in relation to the total transaction, is necessary to reduce the supplier's financial risks in initiating manufacturing. The well-known supply chain trading agent competition (Arunachalam and Sadeh 2005) makes use of a similar cost structure for procurements. Finally, Gurnani and Shi (2006) analyze the issue of supplier reliability under asymmetric beliefs regarding the supplier. One of the two cases analyzed is a down payment contract with overtrust, where the retailer believes that the supplier has a better-than-claimed probability of delivering the order quantity. Under certain regularity conditions, they show that the optimal down-payment is equal to the entire payment to be made to the supplier. We are inspired from this result in our model setting, though the model we come up with also represents the situation where the down payment is not necessarily the entire amount.

A proportional allocation mechanism, among other possibilities, is employed to ration the limited production among the retailers not only because it is conceptually simple and 
easy to implement but also because it is one of the most commonly used mechanisms in practice. Furthermore, it is probably the most intuitive and fair policy since it allocates more to those that order more, that is, those in need. Hence, any retailer may increase its allocation by simply increasing its order quantity, which is the main motivation for these mechanisms to be called as 'manipulable allocation mechanisms'. Linear and proportional allocation are examples of this kind. (See Cachon and Lariviere (1999a, 1999b) for further information on allocation mechanisms.)

In this setting, we consider a generalization of the above when full information on supplier's capacity is not available to some (or all) retailers. If full information is not available to a retailer, then that retailer uses a probability distribution about the capacity of the supplier. We assume that all demand and cost information is common knowledge.

We feel that the rationing game described above has not been properly investigated. Particularly, there are no clear managerial guidelines as to the benefits of a better understanding of the supplier's capacity process. Therefore, the main focus of our inquiry is to answer the following questions:

1. Under what conditions does perfect information provide lower costs, and are there any circumstances where it has a deteriorating effect on cost performance?

2. Assuming that one of the retailers has perfect capacity information, are there any incentives for this retailer to reveal the available information to others?

Most of the studies in the literature assume that information asymmetry exists between different levels of the supply chain and that the asymmetry usually stems from cost or demand parameters or from inventory levels. Moreover, most of the problems are handled via a leader-follower type gaming structure, where one of the echelons is assumed to have the power of offering contracts. Our work differs from existing studies in important aspects. We consider information asymmetry among parties that belong to the same echelon of the supply chain; the information asymmetry regards the capacity of the supplier and retailers are served from the same capacity pool.

Note that we are not concerned with the alignment of individual objectives by a systemwide objective, in other words, a coordination mechanism. Information imperfection is the most apparent characteristic dominating the structure in this environment.

Our main contributions may be summarized as follows:

1. The amount of capacity allocated to each retailer depends not only on its order but also on the sum of the orders of the remaining retailers. We propose the use of a cost structure under which retailers are required to pay the ordering cost (or parts of it) when they place their order. If the quantity they receive is less than their initial order, the difference is refunded at the time of delivery. We show that this cost structure provides an equilibrium point regardless of the conditions on the capacity as long as the lead time is significant. It is also shown to decrease the order amplification of the retailers. Furthermore, we show that the competitive capacity rationing game may lack equilibrium when the lead time is negligible.

2. With minor modifications, the analysis that we perform for the case where lead time is significant is applicable to different settings as well. For instance, if the retailers pay a certain fee for each unit they order regardless of whether they receive it or not, then the resulting analysis is almost identical, even if lead time is negligible. This is also true for the case where each retailer pays a portion of the unit cost for each unit ordered before the allocation and it is not refunded even if its ration is less than its order (see Bakal (2003) for a detailed discussion). Jemai et al. (2010) consider a similar cost structure in a single-supplier, single-retailer context. 
3. We analyze the effects of information asymmetry among the retailers on their ordering decisions and costs, and assess the value of capacity information to the retailers through numerical computations. In our analytical derivations, we model the asymmetry in a general form and let the retailers have estimates of the supplier's probability density function for the capacity. In our computations, however, we restrict ourselves to normal distribution.

The rest of the paper is organized as follows: In Sect. 2, we summarize the related literature and our contribution. Section 3 describes the modeling framework, and Sect. 4 studies the problem analytically. In Sect. 5, we summarize the computational study in order to assess the value of perfect capacity information. We conclude with discussing the results and proposing further research directions in Sect. 6.

\section{Related literature}

The value of information sharing and the effects of information asymmetry among different parties in a supply chain have received a great amount of interest in recent years. With the advances in information technology, firms are now able to keep track of every transaction; this raises the questions of whether to share this information with the other interacting parties or not, and to what extent information sharing should happen. Chen (2003) provides a thorough review of this subject.

Lee and Whang (2000) describes the types of information shared in supply chains and give examples of how information sharing is implemented in different industries. Karaesmen et al. (2004) analyze the value of advance demand information for a single stage, capacitated manufacturing system where the capacity is modelled as a single-server queue. Aviv and Federgruen (1998) study a two-echelon supply chain consisting of a single supplier and multiple retailers where the retailers employ $(m, \beta)$ policy, reviewing their inventory level every $m$ period and raise it to $\beta$. It is observed that centralization and sharing demand information decrease supply chain costs. Gavirneni et al. (1999) and Gavirneni (2002) consider a similar system where the retailer uses an $(s, S)$ type inventory-replenishment policy. Lee et al. (2000) examine a two-echelon supply chain with a single manufacturer and a single retailer. The demand process at the retailer is a simple AR(1) process. Sharing demand information reduces the standard deviation of the lead time demand of the manufacturer and hence lowers the inventory level and expected cost. Cachon and Fisher (2000) investigate the value of inventory information in a one-warehouse multi-retailer system from a centralized perspective where both parties employ $\left(R_{i}, n Q_{i}\right)$ policies. They evaluate the value of information about the retailers' real-time inventory status and conclude that smaller batch sizes and shorter lead times are more valuable to the chain than just sharing the available information.

There are also studies in the literature that address issues such as what mechanisms may be employed to reveal the correct information and how the performance of the supply chain is affected by the implementation of these mechanisms. Lau and Lau (2005) argue that the classical Stackelberg game-type models under deterministic and symmetrical information systems are flawed since gaming is meaningless and locally circular in such systems. They claim that introduction of stochasticity and information asymmetry leads to more plausible models. Corbett and de Groote (2000) consider a two-stage, deterministic lot-sizing problem and investigate the effects of information asymmetry regarding the retailer's holding cost. They primarily show that information asymmetry reduces the efficiency of the system, but that contracting is more efficient than no form of coordination. Ha (2001) considers a 
price-sensitive newsboy model and proves that the centralized solution is no more available when the marginal cost of the buyer is private information. Corbett et al. (2004) also assume a price-sensitive, but linear demand model and conclude that information and flexibility of contracts are strategic complements. Corbett (2001) studies a stochastic two-echelon inventory system including ordering cost. He investigates information asymmetry regarding the ordering cost of the supplier and the backorder cost of the buyer and concludes that the resulting solution is always less efficient than the joint optimum. Lau and Lau (2001) consider information asymmetry regarding demand under a newsboy structure where the manufacturer sets the wholesale price and the retailer selects the order quantity. They show that the effect of information asymmetry depends on whether the manufacturer is aware that the retailer has superior knowledge. Cachon and Lariviere (2001), on the other hand, investigate information asymmetry regarding the initial demand forecast of the manufacturer. A relatively different study comes from Lim (2001), who introduces a contract design problem of a producer purchasing parts from a single supplier in the case of information asymmetry regarding the quality of the parts. Cachon and Zhang (2006) focus on a retailer-driven channel where the supplier can regulate his lead time. They derive a complicated mechanism to minimize the retailer's cost with imperfect information about the supplier's cost and argue that simpler mechanisms are nearly optimal. Wang et al. (2008) consider the interaction between a supplier and a retailer where the retailer possesses private information on market size, market sensitivity, and its own cost. They conclude that it is often not possible for the supplier to induce the retailer to share its information. Wang et al. (2009), considering a retailer-driven channel, complement this study by arguing that a supplier having private information about its own cost does not usually have an incentive to share its information with the retailer. Liu and Çetinkaya (2009) also consider a retailer-driven channel where the supplier has private information about its cost. They study different contract types and conclude that the leadership is not necessarily beneficial under information asymmetry. Li et al. (2009) investigate advance commitment and option contracts when the retailer has superior information about market demand and price. Yang et al. (2009) derive the optimal contract menu offered by a manufacturer to a supplier having private information about supply disruptions.

Lee et al. (1997) consider the rationing concept in a single-period problem where a capacitated supplier serves $N$ identical retailers, employing a proportional allocation mechanism. They show that the optimal order quantity for the retailer exceeds the order quantity in the traditional newsboy problem. Ehrhant (2002) considers the same setting working on two cases: the buyers know the exact capacity vs. the buyers have a prior distribution function. He shows that in both cases the retailers have an incentive to amplify their needs when they expect that capacity will bind. Rong et al. (2008) consider a similar model under perfect (deterministic) and imperfect (stochastic) capacity information, and further assume that the capacity of the supplier is insufficient to cover all retailers' demands. They show that the resulting game does not have an equilibrium. Furthermore, they introduce a reservation payment to alleviate over-ordering in the perfect information case and show that the resulting game has an equilibrium. Their primary purpose is to investigate Bullwhip and Reverse Bullwhip effects under this gaming structure. The work of Rong et al. (2008) is similar to our study in that both papers consider a reservation payment to prevent excessive orders in a rationing game. However, it should be noted that Rong et al. (2008) assume that the retailers are identical and the capacity of the supplier is insufficient to cover all retailers' demands. In our study, we do not impose these assumptions. Furthermore, we investigate the imperfect information model with the reservation payment and investigate the incentives to share perfect information. It should also be noted that the idea of reservation payment to alleviate over-ordering was introduced by Bakal (2003), and forms the basis of our paper as well. 
Cachon and Lariviere (1999a) investigate allocation mechanisms and show that optimal mechanisms are prone to manipulation, whereas truth-telling mechanisms do not maximize retailer profits. Cachon and Lariviere (1999b) consider two independent retailers, each of whom observes its market state privately, and analyze three different allocation mechanisms: Linear, proportional, and uniform allocation. Dai et al. (2005) consider a distribution system with one supplier and two retailers, where customers may go to the other retailer in case of a stockout. They discuss inventory allocation and derive the necessary and sufficient conditions for the existence of a Nash equilibrium. Cachon and Lariviere (1999c), on the other hand, examine a two-period problem where the allocation of a retailer in the second period depends on its sales in the first one. Deshpande and Schwarz (2002) consider a similar setting but take the perspective of the retailer who has private information about its demand. They show that the supplier's capacity choice under information asymmetry is lower than the capacity choice that maximizes centralized profits with full information. The model presented in Mallik and Harker (2004) considers the allocation of the capacity of manufacturing plants among different products having independent demands. They show that both the product and manufacturing plant managers misrepresent their forecasts when the distributions of the demands for the products and capacity for the plants are unknown to the central planner. Schneeweiss and Zimmer (2004) analyze a two-echelon system consisting of a producer and a supplier, each of whom may hold some private information. In their numerical analysis, the effects of private information about the supplier's capacity on the performance of the supply chain are investigated. Note that the model does not include any horizontal competition, hence, capacity allocation issues are not addressed.

Similar mechanisms are also employed in the allocation of aggregate demand among retailers. The studies of Lippman and McCardle (1997), Wang and Gerchak (2001), and Cachon (2003) are examples of such models. Cachon's (2003) work has interesting analogies with our work. First of all, he argues that there may not exist a Nash equilibrium, or there may be multiple Nash equilibria, depending on the wholesale price of the supplier. In our work, when the lead time is negligible, the resulting game possesses the same property, depending on the supply conditions. In Cachon (2003), it is noted that the retailers order more because of the demand-stealing effect, which coincides with order amplification in our case, which retailers do to get a greater share of capacity.

\section{Modeling framework}

We consider a single-period problem where a supplier serves multiple retailers. The retailers are local monopolies and are not in direct competition. There are independent, random demands observed at the retailers. Let $X_{i}$ be the random variable denoting the demand for retailer $i$. At the beginning of the period, all retailers simultaneously announce their order quantities and pay the unit cost for each item they order. Let $z_{i}$ be the order quantity for retailer $i$. The orders are not satisfied immediately as the manufacturer has a production and transportation lead time. The supplier has a restriction on the total amount that can be supplied. Let $S$ be the capacity of the supplier. Note that $S$ is a deterministic quantity. However, a retailer which does not have information on the exact value of $S$ uses an assessed distribution for the supplier's capacity in order to determine its order quantity. After the lead time, if the capacity of the supplier is sufficient to satisfy the total order quantity of the retailers, then each receives its order in full. Otherwise, the supplier allocates the capacity to the retailers proportional to their order quantities. Let $Q=\sum_{i} z_{i}$ be the total order quantity of the retailers, and $Q_{-i}=\sum_{j \neq i} z_{j}$ be the total quantity ordered by retailers other than $i$. 


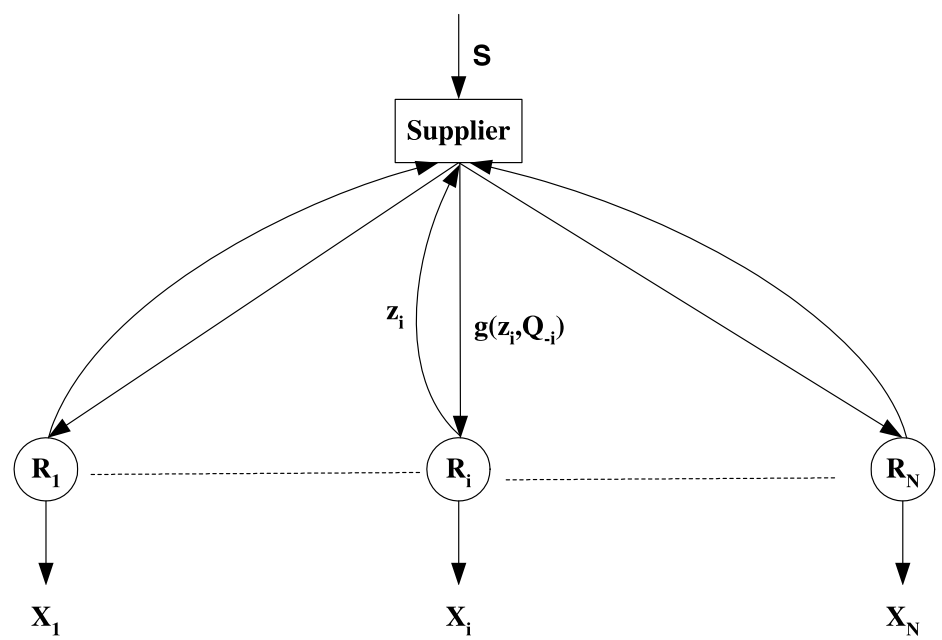

Fig. 1 Problem environment

Then, the quantity allocated to retailer $i$ is given by $g\left(z_{i}, Q_{-i}, S\right)=\min \left(z_{i}, \frac{z_{i} S}{Q}\right)$. Retailers are refunded the unit cost of the items that they ordered but did not receive. After the receipt of the orders, demands at the retailers are realized. Upon satisfying its demand, each retailer incurs relevant costs (see Fig. 1).

There are three factors determining the allocation of a retailer: capacity of the supplier, its individual order and the sum of the orders placed by other retailers. We assume that the retailers do not have initial inventories at the beginning of the period and cannot influence their allocations in any way other than by adjusting order quantities.

Here are the definitions of the variables and parameters used in the model: $X_{i}$

Continuous random variable denoting demand for retailer $i$

$\phi_{i}(x), \Phi_{i}(x) \quad$ Density and distribution function of $X_{i}$

$S \quad$ Capacity of the supplier

$\mathcal{S} \quad$ Random variable denoting the capacity of the supplier assessed by

$f(s), F(s) \quad$ the retailers who do not have perfect in

$z_{i} \quad$ Order quantity for retailer $i$

$Q \quad$ Sum of orders of all retailers, that is $Q=\sum z_{i}$

$Q_{-i} \quad$ Sum of orders of all retailers except retailer $i$, that is $Q_{-i}=\sum_{j \neq i} z_{j}$

$\alpha \quad$ Interest rate for the lead time period

$c^{\prime} \quad$ Unit procurement cost

$h^{\prime} \quad$ Overage cost incurred by the retailer for each unit on hand at the end of the period

$p^{\prime} \quad$ Underage cost incurred by the retailer for each unit of demand unsatisfied

$g\left(z_{i}, Q_{-i}, s\right) \quad$ Amount received by retailer $i$ when it orders $z_{i}$ units, other retailers order a total of $Q_{-i}$ units, and supplier's capacity is $s, g\left(z_{i}, Q_{-i}, s\right)=$ $\min \left(z_{i}, \frac{z_{i} s}{Q}\right)$. 
Given the sum of the order quantities of all other retailers, if full information is available to retailer $i$, it computes its expected discounted cost function as

$$
\begin{aligned}
C_{i}\left(z_{i}, Q_{-i}\right)= & \frac{h^{\prime} E\left[\left(g\left(z_{i}, Q_{-i}, S\right)-X\right)^{+}\right]+p^{\prime} E\left[\left(X-g\left(z_{i}, Q_{-i}, S\right)\right)^{+}\right]}{1+\alpha} \\
& +c^{\prime} z_{i}-\frac{c^{\prime}\left(z_{i}-g\left(z_{i}, Q_{-i}, S\right)\right)}{1+\alpha}
\end{aligned}
$$

where the first term represents the discounted inventory costs, the second term is the initial procurement cost for the entire order, and the last term is the amount received at the end of the period for the portion of the order not delivered. Defining $c=c^{\prime} /(1+\alpha), h=h^{\prime} /(1+$ $\alpha), p=p^{\prime} /(1+\alpha), L(z)=h E\left[(z-X)^{+}\right]+p E\left[(X-z)^{+}\right]+c z$, and rearranging the terms, we get

$$
C_{i}\left(z_{i}, Q_{-i}\right)=L\left(g\left(z_{i}, Q_{-i}, S\right)\right)+c \alpha z_{i} .
$$

On the other hand, if full information is not available, retailer $i$ computes its expected discounted cost function as

$$
C_{i}\left(z_{i}, Q_{-i}\right)=\int_{0}^{\infty} L\left(g\left(z_{i}, Q_{-i}, s\right)\right) d F(s)+c \alpha z_{i} .
$$

In this case, retailer $i$ optimizes its order quantity using (2), which is computed over the perceived capacity. Actual expected cost, on the other hand, should be computed over the true capacity, that is, using (1).

Note that the above characterizations of the cost functions under full information (1) and partial information (2) are valid in a setting where the lead time is negligible and the retailers pay two-part linear costs, first part based on the quantity ordered ( $c \alpha$ per unit) and second part based on the quantity received ( $c$ per unit). Defining $c=c^{\prime}, h=h^{\prime}, p=p^{\prime}$, we get the same cost functions as in (1) and (2). Hence, our analysis in Sect. 4, and our findings through computational analysis in Sect. 5, are valid for such a setting as well.

Given the above framework, we next characterize the rationing game. As we will show in Sect. 4, the game has an equilibrium when the lead time is significant, that is $\alpha>0$, whereas it may lack equilibrium when the lead time is negligible, that is $\alpha=0$.

\section{Analysis of the rationing game}

We first consider the setting where lead time is significant. The retailers pay the unit cost for each unit they order at the time they place their orders. Once the orders are delivered, retailers are refunded the unit cost for the difference between their order and the quantity delivered. However, since lead time is positive, the retailers will have an additional cost (time value), which increases in the order quantity. Hence, the order amplification of the retailers will be less severe when compared to the model with no lead time. It should also be noted that the resulting model also represents the case where the lead time is negligible and the retailers pay a certain fee for each unit they order.

In the following subsections, we analyze the equilibrium under three main cases: (i) All Informed Retailers (all retailers have full information) (ii) All Uninformed Retailers (none of the retailers has full information), and (iii) $K$ Informed Retailer (Only $K$ of the retailers have full information). 


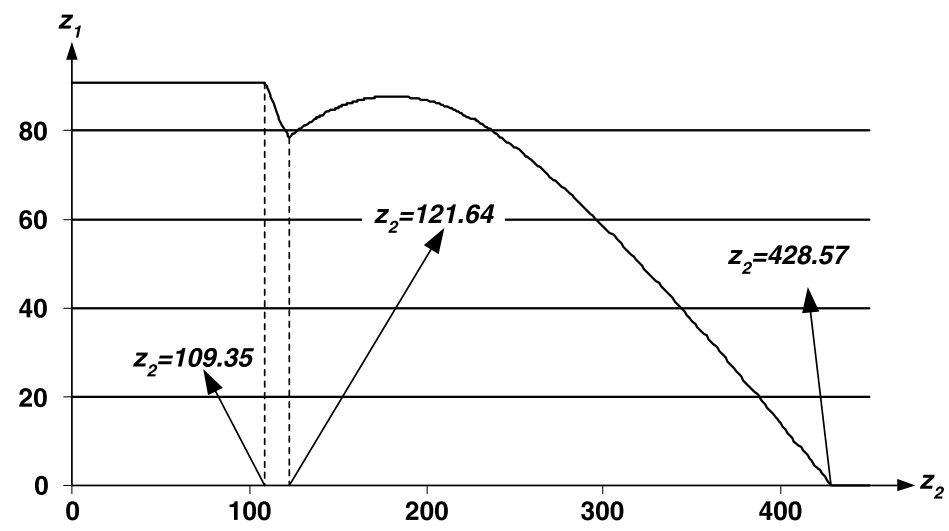

Fig. 2 Response curve for $\left(\alpha=0.7, p=75, c=30, h=0, S=200, \mu_{1}=\mu_{2}=100, \sigma_{1}=\sigma_{2}=20\right.$ )

\subsection{All informed retailers}

With full information, the expected cost function of retailer $i$ is as follows:

$$
C_{i}\left(z_{i}, Q_{-i}\right)=\left\{\begin{array}{ll}
L\left(z_{i}\right)+c z_{i} \alpha & z_{i} \leq S-Q_{-i} \\
L\left(S z_{i} / Q\right)+c z_{i} \alpha & z_{i}>S-Q_{-i}
\end{array} .\right.
$$

Although $C_{i}\left(z_{i}, Q_{-i}\right)$ is pseudo-convex in $z_{i}$, the first order condition on $C_{i}\left(z_{i} Q_{-i}\right)$ is not sufficient to generate the response curve of retailer $i$. However, we can still characterize the response curve utilizing the first order condition:

Proposition 1 The response curve of retailer $i$ is given by

$$
R C_{i}\left(Q_{-i}\right)= \begin{cases}y_{i} & Q_{-i} \leq S-y_{i} \\ S-Q_{-i} & S-y_{i}<Q_{-i} \leq Q_{-i}^{0} \\ \frac{\partial C\left(z_{i}, Q_{-i}\right)}{\partial z_{i}}=0 & Q_{-i}^{0}<Q_{-i} \leq \frac{(p-c) S}{c \alpha} \\ 0 & Q_{-i}>\frac{(p-c) S}{c \alpha}\end{cases}
$$

where $Q_{-i}^{0}$ is the solution to $\left(-p+c+(p+h) \Phi_{i}\left(S-Q_{-i}\right)\right) \frac{Q_{-i}}{S}+c \alpha=0$, and $y_{i}$ is the minimizer of $L(y)+c \alpha y$.

Proof All proofs are provided in the Appendix.

Figure 2 illustrates Proposition 1 for an example setting with two retailers where the demands at the retailers are normally distributed. We observe that retailer $i$ orders and receives its optimal uncapacitated order quantity when the total order quantity of all other retailers is low enough $\left(z_{2}<109.35\right.$ for the example setting). When $S-y_{i}<Q_{-i} \leq Q_{-i}^{0}$ $\left(109.35<z_{2}<121.64\right)$, it cannot get $y_{i}$ units by ordering $y_{i}$. However, rather than increasing its order quantity to get $y_{i}$ units, it decreases its order quantity such that the total orders equal the capacity in order to avoid rationing. Note that there exists a threshold value, $Q_{-i}=\frac{(p-c) S}{c \alpha}\left(z_{2}=428.57\right)$, such that when $Q_{-i}$ exceeds this threshold, retailer $i$ is better off not ordering at all. 
Proposition 2 When there is a positive lead time between orders and deliveries, the rationing game with full information has a Nash equilibrium.

Proof All proofs are provided in the Appendix.

Proposition 2 indicates that when the time value of money is not negligible, the resulting rationing game has an equilibrium point. Furthermore, even if the lead time is negligible, the game still has an equilibrium when the retailers make an initial, nonrefundable payment for each unit they order.

\subsection{All uninformed retailers}

We next consider the case where retailers do not know the capacity of the supplier and use a probability distribution function for it. We assume that the distribution function that each retailer uses is the same, which is a reasonable assumption since the retailers do not have full information; it is reasonable to assume that the information available to generate the estimation of capacity is public. In this setting, the expected cost function perceived by retailer $i$ becomes

$$
C_{i}\left(z_{i}, Q_{-i}\right)=E\left[L\left(g\left(z_{i}, Q_{-i}, s\right)\right)\right]+z_{i} c \alpha .
$$

Retailer $i$ will determine its order quantity using (4). However, it should be noted that the true expected cost is given by (1) once the order quantities are determined.

Unfortunately, we cannot analytically prove that $C_{i}\left(z_{i}, Q_{-i}\right)$ is pseudo-convex. However, through extensive computations using a wide range of parameters and distributions, we conjecture that $C_{i}\left(z_{i}, Q_{-i}\right)$ is pseudo-convex in $z_{i}$.

Proposition 3 If $C_{i}\left(z_{i}, Q_{-i}\right)$ is pseudo-convex in $z_{i}$, then the rationing game where retailers do not have full information on capacity has a Nash equilibrium when lead time is positive.

Proof All proofs are provided in the Appendix.

We may gain further insights into the game by characterizing the response curve of retailer $i$ :

Proposition 4 Let $G\left(Q_{-i}\right)=\left(1-F\left(Q_{-i}\right)\right)+\frac{1}{Q_{-i}} \int_{0}^{Q_{-i}} s d F(s)$. Then, for $Q_{-i}>0$, the response curve of retailer $i, R C_{i}\left(Q_{-i}\right)$, is given by

$$
R C_{i}\left(Q_{-i}\right)=\left\{\begin{array}{ll}
\left\{z_{i}: \frac{\partial C_{i}\left(z_{i}, Q_{-i}\right)}{\partial z_{i}}=0\right\} & Q_{-i} \leq G^{-1}\left(\frac{-c \alpha}{-p+c}\right) \\
0 & Q_{-i}>G^{-1}\left(\frac{-c \alpha}{-p+c}\right)
\end{array} .\right.
$$

Proof All proofs are provided in the Appendix.

Hence, we may conclude that the retailers do not increase their order quantities indefinitely when the lead time is not negligible. On the contrary, they refuse to order when the total amount ordered by other retailers exceeds a threshold value, $Q_{-i}^{0}=G^{-1}\left(\frac{-c \alpha}{-p+c}\right)$.

The exact behaviour of a retailer's reaction would very much depend on cost, demand and capacity parameters (see the Appendix for the analysis of the response curve). This is mainly because of the tradeoff between underage/overage costs and the time value of money 


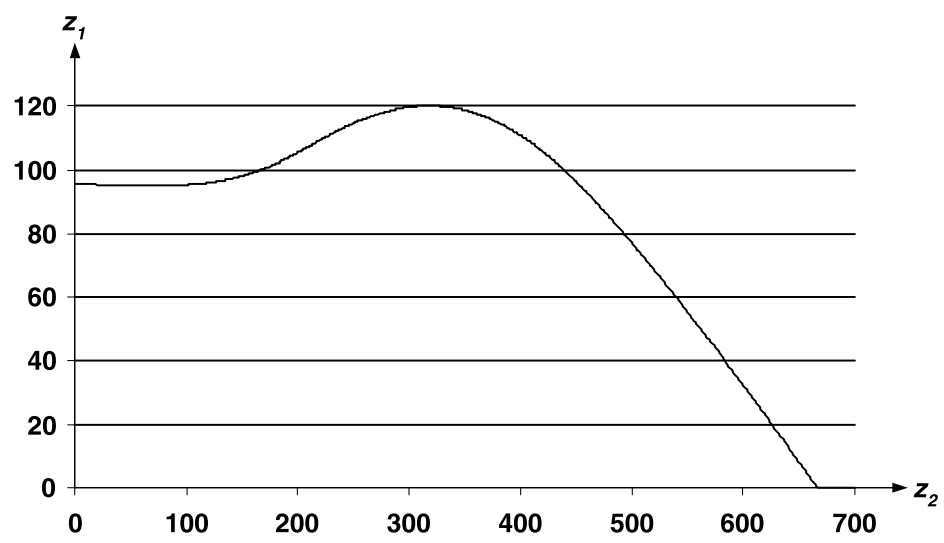

Fig. 3 Response curve for $\left(\alpha=0.45, p=75, c=30, h=0, \mu_{\mathcal{S}}=200, \sigma_{\mathcal{S}}=200 / 3, \mu_{1}=\mu_{2}=100\right.$, $\left.\sigma_{1}=\sigma_{2}=20\right)$

due to the lead time. Assume that $z_{i}^{*}$ is the optimal response of retailer $i$ to a given $Q_{-i}$. When $Q_{-i}$ increases, $z_{i}^{*}$ is no longer optimal for retailer $i$ since capacity allocated to this retailer decreases if its order quantity remains $z_{i}^{*}$. Hence, an increase in $Q_{-i}$ affects the costs of retailer $i$ in two ways: expected underage cost incurred increases, while overage cost decreases. By increasing its order quantity, the retailer may reduce the effects of increasing underage costs. However, because of the lead time, increasing the order quantity increases the cost due to the time value of money as well. Figure 3 illustrates the optimal response of a retailer to the order quantity of the other retailer when the retailer's assessment for the distribution of capacity is normal with mean $\mu_{\mathcal{S}}=200$ and standard deviation $\sigma_{\mathcal{S}}=200 / 3$. In this example setting, Retailer 1 increases its order quantity until $z_{2}=318.14$. However, after this threshold value, the increases in the costs of time value and overage outweigh the decrease in underage cost, as a result of an increase in $z_{1}$. Hence, Retailer 1 starts to decrease its order quantity when $z_{2}$ increases beyond the threshold value. When $z_{2}$ hits 666.69 , Retailer 1 is better off not ordering at all.

\section{$4.3 K$ informed retailers}

We start considering a special case where only one of the retailers, Retailer 1, has full information on the capacity of the supplier $(K=1)$. Other retailers do not know that Retailer 1 has full information and they share the same prediction of supply, in other words, use the same probability distribution of supply. In such a case, all retailers but Retailer 1 will determine their order quantities as characterized in the case where none of the retailers has full information, given in Sect. 4.2. That is, the retailers other than Retailer 1 will determine their order quantities using (4) and assuming that all other retailers use the same capacity distribution. On the other hand, Retailer 1 will be able to determine the order quantities of the other retailers beforehand by characterizing their equilibrium behavior, and arrange its own order quantity accordingly, utilizing the full information and using (3).

We illustrate the above discussion for an example data set in Fig. 4. In this example, Retailer 2 does not have perfect information and estimates that the capacity is normally distributed with mean 200 and standard deviation 200/3. It also assumes that Retailer 1 operates using the same distribution. However, Retailer 1 has perfect capacity information that $S=200$. Retailer 2 determines the equilibrium point using (5), which is $\left(z_{1}, z_{2}\right)=$ 


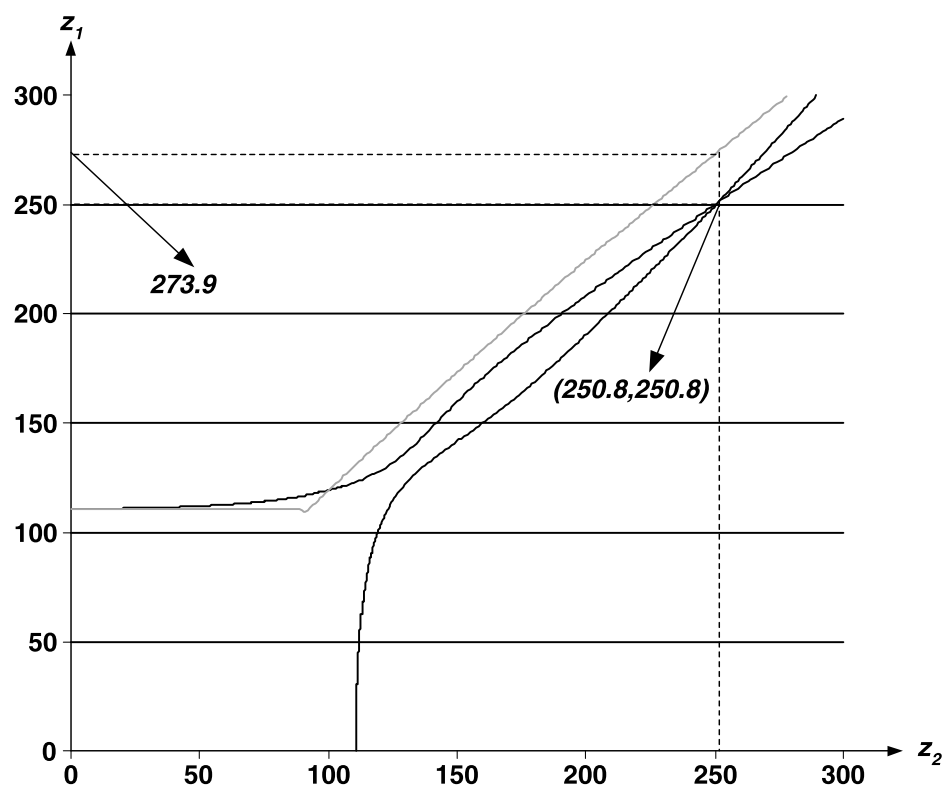

Fig. 4 Only retailer 1 has perfect information: $\left(\alpha=0.1, p=75, c=20, h=0, \mu_{\mathcal{S}}=200, \sigma_{\mathcal{S}}=200 / 3\right.$, $S=200, \mu_{1}=\mu_{2}=100, \sigma_{1}=\sigma_{2}=20$ )

(250.82, 250.82). Hence, the optimal order quantity of Retailer 2 is 250.82 . Retailer 1 also characterizes this equilibrium and hence determines the order quantity of Retailer 2 . Its response curve is characterized by (3) (the grey curve in the figure). Given the order of Retailer 2, the optimal order quantity for Retailer 1 is 273.88 .

The arguments above can be extended to the case where a group of retailers has perfect information. Let $A$ and $B$ denote the set of retailers with and without perfect information, respectively. Then, retailer $j \in B$ will determine its order quantity assuming that all retailers lack perfect information, that is, by solving for the equilibrium of the game where all retailers lack perfect information. If each retailer $i \in A$ believes that all other retailers (even those in $A$ ) lack perfect information, then it determines the equilibrium of the aforementioned game, and assuming that all other retailers will operate at that point, it characterizes its optimal order quantity as in Proposition 1 . If every retailer $i \in A$ is aware that all retailers in $A$ have perfect information, its optimal order quantity is determined as follows: Retailer $i$ knows that any retailer $j \in B$ operates at the aforementioned equilibrium. Given their order quantities and being aware that any retailer in $A$ has the same information, it solves for the equilibrium among the retailers in $A$ characterized by the response curves provided in Proposition 1.

We illustrate the above discussion for a three-retailer system where Retailer 1 and Retailer 2 have perfect information, whereas Retailer 3 does not. The parameters used in the example are $\left(\alpha=0.1, p=75, c=20, h=0, \mu_{\mathcal{S}}=350, \sigma_{\mathcal{S}}=70, S=300, \mu_{1}=\mu_{2}=\mu_{2}=\right.$ $100, \sigma_{1}=\sigma_{2}=\sigma_{2}=20$ ). We focus on the optimal order quantity of Retailer 1 . Retailer 3 , being unaware that the other retailers have perfect information, obtains the equilibrium point assuming that the other retailers use the same capacity distribution. Its optimal order quantity is 117.1 and it expects the other retailers to place the same order (since all retailers are identical in the example). Retailer 1 and Retailer 2 are aware that Retailer 3 will place an order of 117.1. If Retailer 1 is not aware that Retailer 2 has perfect information and vice 
versa, then it believes that Retailer 2 will place an order of size 117.1 and optimizes its own order accordingly. The optimal order size is 134.5 . On the other hand, if it is aware that Retailer 2 also has perfect information, its order quantity is 155.6 .

\subsection{Negligible lead time}

In this section, we briefly comment on the setting where lead time is negligible $(\alpha=0)$. The reader may refer to Bakal (2003) for a thorough analysis of this setting.

When all retailers have full information about the capacity of the supplier, the resulting rationing game does not have an equilibrium point if the capacity of the supplier, $S$, is smaller than $\sum y_{i}$. When $S>\sum y_{i}$, the game has a unique equilibrium. When $S=\sum y_{i}$, the game has an infinite number of equilibria.

When none of the retailers has full information about the capacity of the supplier, since $\lim _{z_{i} \rightarrow \infty} g\left(z_{i}, Q_{-i}, S\right)=S$, the expected cost function reduces to $\lim _{z_{i} \rightarrow \infty} C_{i}\left(z_{i}, Q_{-i}\right)=$ $E[L(\mathcal{S})]$, which is finite for most of the distributions. Noting that the strategy space for the rationing game is unbounded, we may conclude that sufficient conditions for a Nash equilibrium to exist are not satisfied and the model may lack a Nash equilibrium. When the upper bound on the support of the distribution function, $\bar{S}$, is lower than the sum of the newsboy order quantities of the retailers, that is, $\sum y_{i}$, the resulting game does not have a Nash equilibrium point. When the lower bound, $\underline{S}$, is greater than $\sum y_{i}$, the resulting game has a unique Nash equilibrium point. For the case where $\underline{S} \leq \sum y_{i} \leq \bar{S}$, we cannot directly comment on the existence and uniqueness of the Nash equilibrium. However, extensive calculations and analysis performed using different parameters and capacity distributions show that, given all parameters and density functions, there exist $m_{1}$ and $m_{2}$ such that

- when $E[\mathcal{S}]<m_{1}$, the rationing game does not have a Nash equilibrium.

- when $m_{1}<E[\mathcal{S}]<m_{2}$, there are two Nash equilibria.

- when $E[\mathcal{S}]>m_{2}$, there is a unique Nash equilibrium.

The case where $K$ of the retailers have perfect information can be analyzed as in Sect. 4.3.

\section{Computational analysis}

In this section our aim is to analyze how order quantities and expected costs of the retailers react to changes in problem parameters, and to provide managerial insights regarding the questions posed in Sect. 1 by computationally analyzing (i) benefits of capacity information, and (ii) incentives to share information. For these purposes, we restrict the number of retailers to two for computational tractability (denoted by $R_{1}$ and $R_{2}$ ), and we consider three different settings: (i) Both retailers are informed (Both Informed), (ii) Both retailers are uninformed (Both Uninformed), and (iii) Only one retailer is informed (Only $R_{i}$ is Informed). Note that the first two cases represent supply information symmetry among retailers, whereas the third one represents information asymmetry.

The expected costs of the retailers will be calculated using the true capacity of the supplier once their order quantities are determined. It should also be noted that the costs are calculated at the time orders are delivered. The demand observed by $R_{i}$ is a normal random variable with mean $\mu_{i}$ and coefficient of variation $c v_{i}$. 
Table 1 Data set for the computational study

\begin{tabular}{rllllllllll}
\hline$\alpha$ & $p$ & $h$ & $c$ & $\mu_{1}$ & $\mu_{2}$ & $c v_{1}$ & $c v_{2}$ & $r$ & $b$ & $c v_{s}$ \\
\hline 0.1 & 60 & 0 & 20 & 80 & 100 & 0.1 & 0.2 & 1 & 1 & 0.1 \\
& 75 & & & 100 & & 0.2 & & 1.2 & 1.2 & 0.2 \\
& 90 & & & 120 & & 0.3 & & & & 0.3 \\
\hline
\end{tabular}

Table 2 Value of information: demonstrative examples, both uninformed vs both informed

\begin{tabular}{|c|c|c|c|c|c|c|c|c|c|c|}
\hline \multirow[t]{2}{*}{$r$} & \multirow[t]{2}{*}{$b$} & \multicolumn{4}{|c|}{ Perfect information } & \multicolumn{4}{|c|}{ No information } & \multirow[t]{2}{*}{$\%$ Gain } \\
\hline & & $\overline{z_{1}}$ & $z_{2}$ & $g\left(z_{1}, z_{2}\right)$ & $g\left(z_{2}, z_{1}\right)$ & $z_{1}$ & $z_{2}$ & $g\left(z_{1}, z_{2}\right)$ & $g\left(z_{2}, z_{1}\right)$ & \\
\hline 1.2 & 1 & 113.8 & 113.8 & 113.8 & 113.8 & 498.1 & 498.1 & 120 & 120 & 22.0 \\
\hline 1 & 1 & 625 & 625 & 100 & 100 & 498.1 & 498.1 & 100 & 100 & -6.8 \\
\hline 1 & 1.2 & 625 & 625 & 100 & 100 & 118.7 & 118.7 & 100 & 100 & -34.3 \\
\hline 1.2 & 1.2 & 113.8 & 113.8 & 113.8 & 113.8 & 118.7 & 118.7 & 118.7 & 118.7 & 0.56 \\
\hline
\end{tabular}

\subsection{Value of perfect information}

In this section, we aim to quantify the value of perfect capacity information from the perspective of the first retailer. For this matter, we perform the following comparisons: (i) Both Uninformed versus Both Informed, which quantifies the value of perfect information to $R_{1}$ when information is available to both retailers, (ii) Both Uninformed versus Only $R_{1}$ is Informed, which considers the value of information when only $R_{1}$ gets to know the exact capacity, and (iii) Only $R_{2}$ is Informed versus Both Informed, which measures the value of information to $R_{1}$ when $R_{2}$ already has the information. In case (i), we assume that information is made public, that is, each retailer gets perfect information, and is aware that the other retailer has perfect information as well. On the other hand, in cases (ii) and (iii), the retailer that has perfect information receives it privately. Hence, it assumes that the other retailer does not have perfect information. Table 1 gives the parameter values used for computational analysis. Note that the capacity of the supplier is given by $\left(S=r \times\left(\mu_{1}+\mu_{2}\right)\right)$. Hence, $r=1$ corresponds to the case where the actual capacity is insufficient to cover the needs of the retailers, whereas $r=1.2$ indicates that it is sufficient.

We also provide summaries of the results for a number of examples to illustrate the change in order quantities and expected costs due to perfect information. Common parameters used in all such examples are $\alpha=0.1, p=90, h=0, c=20, \mu_{1}=\mu_{2}=100, c v_{1}=$ $c v_{2}=0.2$, and $c v_{s}=0.2$. Recall that $z_{i}$ and $g\left(z_{i}, z_{j}\right)$ denote the order quantity and final allocation of retailer $i$, respectively. '\%Gain' columns in the corresponding tables depict the improvements in expected cost due to perfect information, that is,

$$
\% \text { Gain }=\frac{C_{1}\left(z_{1}, z_{2}\right) \text { without perfect information }-C_{1}\left(z_{1}, z_{2}\right) \text { with perfect information }}{C_{1}\left(z_{1}, z_{2}\right) \text { without perfect information }} .
$$

\subsubsection{Both uninformed versus both informed}

We start with the analysis of the value of information to $R_{1}$ when it is available to both parties. Table 2 provides demonstrative examples for this setting under different $r$ and $b$ values. Note that $r=S /\left(\mu_{1}+\mu_{2}\right)$, and $b=E[\mathcal{S}] /\left(\mu_{1}+\mu_{2}\right)$. 
We first consider the case where the retailers estimate the capacity to be insufficient $(b=1)$. In this case, order quantities are much higher than what they will actually get, especially when $p$ is high. If actual capacity is ample $(r=1.2)$, and capacity information is revealed to both retailers, the expected costs of the retailers always decrease, since perfect information results in reduced order quantities (see Table 2, Row 1 for an example). Reduced orders benefit the retailers in two aspects: (i) the loss due to the time value of money decreases since less is paid in advance, and (ii) expected excess inventory decreases. The average decrease in the expected cost of $R_{1}$ is $14.6 \%$. Note that the decrease will be higher if actual capacity is larger as this will cause excess inventory to be higher when the retailers do not have perfect capacity information. If the coefficient of variation of capacity is higher, that is, if the retailers perceive capacity as more variable, the benefits of information is lower since higher uncertainty decreases the order quantities. Similarly, when $p$ gets higher, the benefits get more significant. If actual capacity is insufficient $(r=1)$ and retailers estimate the capacity to be insufficient as well $(b=1)$, then perfect information always results in a loss for the retailers. This is because uncertainty has a decreasing effect on the order quantities of the retailers. The allocations are almost the same regardless of the level of information (see Table 2, Row 2 for an example). Hence, perfect information increases the degree of competition between the retailers for the capacity and increases order quantities, which results in higher costs. In this case, as the retailers' perception of capacity becomes more uncertain, the percentage loss from perfect information increases as well.

We now consider the case where the retailers estimate that mean capacity is sufficient to cover their needs. In this case, the order quantities of the retailers are close to their actual needs since they do not engage in a fierce competition for capacity. Hence, if actual capacity is insufficient, the capacity will be rationed between the retailers according to their needs when they do not have perfect information. On the other hand, when capacity information is revealed, the retailers will compete for the insufficient capacity and increase their order quantities. Their allocations will remain almost the same as in the no-information case, resulting in increased costs. Hence, we may deduce that perfect information will increase the costs of the retailers (see Table 2, Row 3). The increase is $22 \%$ on the average for the data set we consider. On the other hand, if actual capacity is sufficient as the retailers estimate it is, perfect information always benefits the retailers (an example: Table 2, Row 4). However, the gain is insignificant, $0.27 \%$ on the average.

To sum up, when information is available to both parties, its value depends on the level of actual capacity. If actual capacity is sufficient to cover the retailers' needs, it is always beneficial. If the retailers believe that capacity was insufficient, the gain with information can be as high as $30 \%$. Otherwise, perfect information increases the degree of competition for capacity, which hurts the retailers, especially if the retailers estimate the capacity to be sufficient. In that case, the loss can be as high as $36.7 \%$.

\subsubsection{Both uninformed versus only $R_{1}$ is informed}

We now consider the case where perfect information is only available to $R_{1}$ (see Table 3 for demonstrative examples). In this case, perfect information always benefits $R_{1}$. The value of information is most significant when actual capacity is sufficient, but the retailers estimate that it is scarce; the maximum and average gains are $11.25 \%$ and $5.07 \%$, respectively. In such a case, perfect information prevents $R_{1}$ from placing an unnecessarily large order, which would result in higher costs due to advance payment and excess inventory (for instance, Table 3, Row 1). Percentage gain decreases as the uncertainty in the retailers' estimates increase. The gain is still significant when actual capacity is insufficient, but retailers expect 
Table 3 Value of information: demonstrative examples, both uninformed vs only $R_{1}$ is informed

\begin{tabular}{|c|c|c|c|c|c|c|c|c|c|c|}
\hline \multirow[t]{2}{*}{$r$} & \multirow[t]{2}{*}{$b$} & \multicolumn{4}{|c|}{ Perfect information } & \multicolumn{4}{|c|}{ No information } & \multirow[t]{2}{*}{$\%$ Gain } \\
\hline & & $z_{1}$ & $z_{2}$ & $g\left(z_{1}, z_{2}\right)$ & $g\left(z_{2}, z_{1}\right)$ & $z_{1}$ & $z_{2}$ & $g\left(z_{1}, z_{2}\right)$ & $g\left(z_{2}, z_{1}\right)$ & \\
\hline 1.2 & 1 & 397.7 & 498.1 & 106.6 & 133.4 & 498.1 & 498.1 & 120 & 120 & 4.5 \\
\hline 1 & 1 & 521.54 & 498.1 & 102.3 & 97.7 & 498.1 & 498.1 & 100 & 100 & 0.16 \\
\hline 1 & 1.2 & 148.4 & 118.7 & 111.1 & 88.9 & 118.7 & 118.7 & 100 & 100 & 3.7 \\
\hline 1.2 & 1.2 & 113.8 & 118.7 & 113.8 & 118.7 & 118.7 & 118.7 & 118.7 & 118.7 & 0.56 \\
\hline
\end{tabular}

Table 4 Value of information: demonstrative examples, only $R_{2}$ is informed vs both informed retailers

\begin{tabular}{|c|c|c|c|c|c|c|c|c|c|c|}
\hline \multirow[t]{2}{*}{$r$} & \multirow[t]{2}{*}{$b$} & \multicolumn{4}{|c|}{ Perfect information } & \multicolumn{4}{|c|}{ No information } & \multirow[t]{2}{*}{$\%$ Gain } \\
\hline & & $z_{1}$ & $z_{2}$ & $g\left(z_{1}, z_{2}\right)$ & $g\left(z_{2}, z_{1}\right)$ & $z_{1}$ & $z_{2}$ & $g\left(z_{1}, z_{2}\right)$ & $g\left(z_{2}, z_{1}\right)$ & \\
\hline 1.2 & 1 & 397.7 & 397.7 & 120 & 120 & 498.1 & 397.7 & 133.4 & 106.6 & 9.6 \\
\hline 1 & 1 & 521.5 & 521.5 & 100 & 100 & 498.1 & 521.5 & 97.7 & 102.3 & 0.41 \\
\hline 1 & 1.2 & 148.4 & 148.4 & 100 & 100 & 118.7 & 148.4 & 88.9 & 111.1 & 9.8 \\
\hline 1.2 & 1.2 & 113.8 & 113.8 & 113.8 & 113.8 & 118.7 & 113.8 & 118.7 & 113.8 & 0.56 \\
\hline
\end{tabular}

Table $5 \%$ decrease in costs due to perfect information over all combinations of parameters

\begin{tabular}{lcccc}
\hline & \multicolumn{4}{l}{ Actual capacity-Average capacity Estimate } \\
\cline { 2 - 4 } & Scarce-Scarce & Ample-Ample & Ample-Scarce & Scarce-Ample \\
\hline Both versus Neither & -6.7 & 0.3 & 14.6 & -22.0 \\
Only $R_{1}$ versus Neither & 0.2 & 0.3 & 5.1 & 2.2 \\
Both versus Only $R_{2}$ & 0.6 & 0.3 & 8.9 & 5.7 \\
\hline
\end{tabular}

it to be sufficient (maximum and average, $5.57 \%$ and $2.23 \%$, respectively). In this case, $R_{2}$, thinking that there is not a competition for capacity, places an order close to its actual need. On the other hand, $R_{1}$, having the information that capacity is scarce, will order more to get a greater share of the limited capacity (see Table 3, Row 3). When actual capacity and estimates are both ample/scarce, the gain due to perfect information is almost negligible.

\subsubsection{Only $R_{2}$ is informed versus both informed}

We now quantify the value of perfect information to $R_{1}$ when $R_{2}$ has perfect information (see Table 4 for examples). Note that $R_{1}$ is not aware that $R_{2}$ has perfect information. Similarly, when $R_{1}$ acquires capacity information, $R_{2}$ is not aware either.

We observe that perfect information always benefits $R_{1}$. The benefits are substantial when the mean estimate is scarce, but actual capacity is sufficient. In this case, the average and maximum decreases in the expected costs of $R_{1}$ are $8.93 \%$ and $16.09 \%$, respectively. Savings with perfect information decreases in the coefficient of variation of capacity. The benefits are still significant when the actual capacity is scarce and the mean estimate is sufficient. However, when both actual capacity and retailers' estimates are scarce/ample, perfect information does not lead to a significant decrease in $R_{1}$ 's expected costs.

Table 5 summarizes our findings on the value of perfect information over all combinations of parameters presented in Table 1 . Note that if limited information leads retailers to 
estimate an ample capacity, there is not much incentive for $R_{1}$ to seek perfect information as the gain would be insignificant in most cases. Furthermore, if actual capacity turns out to be scarce and this information is revealed to both retailers, the expected cost of $R_{1}$ increases considerably. On the other hand, if $R_{1}$ believes that capacity is scarce, then it is better to seek perfect information. If actual capacity turns out to be ample, the gain will be substantial. However, $R_{1}$ should also be aware that if actual capacity is scarce as expected and $R_{2}$ also knows it, its resulting expected cost will be much higher when compared to the case without perfect information.

\subsection{Incentives on information sharing}

In this section, we identify the cases where $R_{1}$ has an incentive to share the perfect information with $R_{2}$ and where it is not beneficial for $R_{1}$ to reveal it. Note that we do not investigate the mechanisms to induce truthful information sharing; the design of such mechanisms that would achieve this is beyond the scope of this study. Instead, we compare the following circumstances and identify when it is beneficial for $R_{1}$ to reveal the information: (Only $R_{1}$ has perfect information) vs. (Both have perfect information). Note that when only $R_{1}$ has perfect information, $R_{2}$ does not know that $R_{1}$ has it. However, when $R_{1}$ shares the information with $R_{2}$, both parties know that the other party has perfect information.

If capacity is insufficient to cover the demand, $R_{1}$ is better off not sharing the capacity information. Even if $R_{2}$ correctly estimates it to be scarce, without perfect information, $R_{2}$ operates under uncertainty, which prevents it from increasing its order quantity as much as in the deterministic case. Since perfect information will cause $R_{2}$ to increase its order, $R_{1}$ will have to increase its order as well to maintain its allocation, which results in an increase in expected costs. Maximum and average increases in the expected cost of $R_{1}$ are $15.54 \%$ and $6.92 \%$, respectively. The loss due to information sharing increases if the coefficient of variation increases. The increase in the cost of $R_{1}$ due to information sharing is even higher if $R_{2}$ 's estimate indicates a sufficient capacity, but actual capacity is scarce. In this case, without perfect information, the order quantity of $R_{2}$ is close to its need. Being aware of actual capacity, $R_{1}$ need not increase its order quantity too much to get the allocation it wants. However, information sharing will cause $R_{2}$ to increase its order, which prevents $R_{1}$ from securing its desired level of allocation with a relatively small order. In this case, the increase in the expected cost of $R_{1}$ can be as high as $41.4 \%$, the average being $24.91 \%$.

If actual capacity is sufficient, $R_{1}$ has an incentive to share perfect information. Although the benefit is negligible when the estimate of $R_{2}$ is also sufficient, it can be as high as $21.82 \%$ when the estimate is scarce. The average gain due to sharing is $10.09 \%$ in this case. The benefits increase in $c v_{s}$ and $p$.

\section{Conclusion}

In the literature, there are few studies that analyze a capacitated model where multiple retailers compete for limited capacity. Lee et al. (1997) consider a single-period problem with capacitated supply and show that order quantities of the retailers are amplified when the supplier has a capacity constraint and proportional allocation mechanism is employed. However, the existence and uniqueness of a Nash equilibrium are not addressed. Also, the magnitude of the amplification under different settings is not considered. Interestingly, there is no study in the literature that investigates the value of perfect capacity information to the retailers.

In this study, we modify the "rationing game" described in Lee et al. (1997) such that the retailers pay the procurement cost when they place their orders, and they are refunded for 
the amount that they do not receive due to capacity restriction. In this setting, we show that given cost and demand parameters, if the lead time between order placement and delivery is negligible, the resulting game may lack equilibrium, may have multiple equilibria, or may have a unique equilibrium, depending on the capacity parameters. The non-equilibrium case occurs when the capacity of the supplier is too limited to satisfy the orders. In this case, the amplification of retailer orders is so severe that an equilibrium point does not exist. On the other hand, if the lead time is greater than zero such that time value of money during the lead time matters, we prove the existence of an equilibrium. These discussions are also valid in a setting where there is no lead time but the retailers pay a two-part linear cost, the first part based on the quantity ordered and the second part based on the quantity received. In such a setting, a positive payment on quantity ordered corresponds to a positive lead time, and no payment on quantity ordered coincides with a negligible lead time. We then try to observe the effects of information asymmetry about the capacity conditions under different settings and assess the value of information. Particularly, we conclude that perfect information is beneficial to retailers when it enables them to decrease their order quantities under tight capacity cases. We also observed that perfect capacity information may have a detrimental effect on retailers' costs when actual capacity is scarce and information is made available to all parties. Upon analyzing incentives to share information, we observe that the retailer with perfect information is reluctant to reveal the information when capacity is scarce, whereas information sharing provides considerable benefits when the capacity is ample.

One of the possible extensions to this study is to include the supplier as a player in the analysis. Recall that the unit cost of the item and the capacity are determined exogenously in our model. When the supplier is included in the analysis as trying to maximize his profit, these parameters may be used as decision variables and a contracting scheme may be generated upon them. However, this approach may complicate the analysis significantly and hence some simplifying assumptions may be needed. There may also be extensions where the problem is addressed in a multi-period setting. The retailers may update their initial estimates of capacity according to the realizations of it. However, the analysis of that problem may be too difficult to handle in such a setting.

\section{Appendix}

Proof of Proposition 1

The derivative of $C\left(z_{i}, Q_{-i}\right)$ with respect to $z_{i}$ is given by

$$
\frac{\partial C\left(z_{i}, Q_{-i}\right)}{\partial z_{i}}=\left\{\begin{array}{ll}
-p+c+(p+h) \Phi_{i}\left(z_{i}\right)+c \alpha & z_{i}<S-Q_{-i} \\
\left(-p+c+(p+h) \Phi_{i}\left(\frac{S z_{i}}{Q}\right)\right) S \frac{Q-z_{i}}{Q^{2}}+c \alpha & z_{i}>S-Q_{-i}
\end{array} .\right.
$$

When $Q_{-i}=0$, equating the derivative of the cost function to zero yields the response of Retailer 1 , which is $z_{i}=y_{i}$. This is the case until $Q_{-i}=S-y_{i}$, where the sum of the orders equals the capacity. When $Q_{-i}=S-y_{i}$, the cost-minimizing $z_{i}$ will be $y_{i}$ and the minimum of the cost function will occur when $Q=S$, the breakpoint, and the derivative of the first part is equal to zero. At this point, the derivative of the second part is greater than zero. When we increase $Q_{-i}$, the derivative of the first part will be negative when $Q=S$ since $z_{i}<y_{i}$. The derivative of the second part will continue to be positive until $Q_{-i}=Q_{-i}^{0}$, implying that the minimum occurs at $z_{i}=S-Q_{-i}$, where neither of the derivatives is zero. Therefore for $Q_{-i}$ values between $S-y_{i}$ and $Q_{-i}^{0}$, neither of the derivatives is zero and the 
minimum of the cost function occurs at $z_{i}=S-Q_{-i}$. After this point, the minimum of the cost function occurs when $Q>S$ and thus the derivative of the cost function characterizes the response curve until $Q_{-i}=\frac{(p-c) S}{c \alpha}$. From this point on, the derivative is always positive, indicating that it is optimal for retailer $i$ not to order at all.

Proof of Proposition 2

The proposition directly follows from Theorem 4.3 and Corollary 4.2 of Basar and Oldser (1995).

\section{Proof of Proposition 3}

The proposition directly follows from Theorem 4.3 and Corollary 4.2 of Basar and Oldser (1995).

\section{Proof of Proposition 4}

$\frac{d G\left(Q_{-i}\right)}{d Q_{-i}}=-\frac{1}{Q_{-i}^{2}} \int_{0}^{Q_{-i}} s d F(s)<0$. Hence, $G\left(Q_{-i}\right)$ is strictly decreasing and invertible. Response curve of retailer $i$ is defined as the set of cost-minimizing order levels of retailer $i$, given $Q_{-i}$. For $Q_{-i} \leq G^{-1}\left(\frac{-c \alpha}{-p+c}\right)$, that is, $G\left(Q_{-i}\right) \geq \frac{-c \alpha}{-p+c}, \frac{d C_{i}\left(z_{i}\right)}{d z_{i}}$ has a unique solution. Since $C_{i}\left(z_{i}\right)$ is pseudo-convex, the unique solution is the cost-minimizing point. For $Q_{-i}>G^{-1}\left(\frac{-c \alpha}{-p+c}\right)$, that is, $G\left(Q_{-i}\right)<\frac{-c \alpha}{-p+c}, C_{i}\left(z_{i}\right)$ is strictly increasing. Because of pseudo-convexity, it is minimized at the lower bound of $z_{i}$, which is 0 .

Behaviour of the response curve

$$
\frac{d R C_{i}\left(Q_{-i}\right)}{d Q_{-i}}=\frac{d\left(d C_{i}\left(z_{i}\right) / d z_{i}=0\right)}{d z_{i}}=\frac{-\partial^{2} C_{i}\left(z_{i}, Q_{-i}\right) / \partial z_{i} \partial Q_{-i}}{\partial^{2} C_{i}\left(z_{i}, Q_{-i}\right) / \partial z_{i}^{2}}=\frac{P_{1}\left(z_{i}, Q_{-i}\right)}{P_{2}\left(z_{i}, Q_{-i}\right)},
$$

where

$$
\begin{aligned}
P_{1}\left(z_{i}, Q_{-i}\right)= & \frac{z_{i} f(Q)}{Q}\left[-p+c+(p+h) \Phi_{i}\left(z_{i}\right)\right]+\int_{0}^{Q} \frac{s z_{i} Q_{-i}}{Q^{4}}(p+h) \phi_{i}\left(\frac{s z_{i}}{Q}\right) s d F(s) \\
& +\int_{0}^{Q} \frac{2 Q_{-i}-Q}{Q^{3}}\left(-p+c+(p+h) \Phi_{i}\left(\frac{s z_{i}}{Q}\right)\right) s d F(s), \\
P_{2}\left(z_{i}, Q_{-i}\right)= & -\frac{z_{i} f(Q)}{Q}\left[-p+c+(p+h) \Phi_{i}\left(z_{i}\right)\right]+(p+h) \phi_{i}\left(z_{i}\right)(1-F(Q)) \\
& +\int_{0}^{Q} \frac{s Q_{-i}^{2}}{Q^{4}}(p+h) \phi_{i}\left(\frac{s z_{i}}{Q}\right) s d F(s) \\
& -\int_{0}^{Q} \frac{2 Q_{-i}}{Q^{3}}\left(-p+c+(p+h) \Phi_{i}\left(\frac{s z_{i}}{Q}\right)\right) s d F(s) .
\end{aligned}
$$

$P_{2}\left(z_{i}, Q_{-i}\right)$ is positive at the points on the response curve since the cost function is pseudoconvex. Hence, in order to comment on the behavior of $R C_{i}\left(Q_{-i}\right)$ with respect to a change in $Q_{-i}$, it is sufficient to analyze $P_{1}\left(z_{i}, Q_{-i}\right)$. 
Recall that retailer $i$ has a positive response when $Q_{-i}=0$ and there exists a $Q_{-i}^{0}$ value beyond which it does not order at all. Let us first analyze the behavior of $R C_{i}\left(Q_{-i}\right)$ at $\left(z_{i}, Q_{-i}\right)=\left(0, Q_{-i}^{0}\right)$. At this point,

$$
P_{1}\left(0, Q_{-i}^{0}\right)=\int_{0}^{Q_{-i}^{0}}-\frac{p-c}{\left(Q_{-i}^{0}\right)^{2}} s d F(s)<0 .
$$

Therefore, we may conclude that $R C_{i}\left(Q_{-i}\right)$ is decreasing when $Q_{-i}=Q_{-i}^{0}$. Now consider the case where $Q_{-i}=0$. At this point,

$$
P_{1}\left(z_{i}, 0\right)=f\left(z_{i}\right)\left[-p+c+(p+h) \Phi_{i}\left(z_{i}\right)\right]-\int_{0}^{z_{i}} \frac{1}{z_{i}^{2}}\left[-p+c+(p+h) \Phi_{i}(s)\right] s d F(s) .
$$

Note that the first order condition for the point $\left(z_{i}, Q_{-i}\right)=\left(z_{i}, 0\right)$ is

$$
\left[1-F\left(z_{i}\right)\right]\left[-p+c+(p+h) \Phi_{i}\left(z_{i}\right)\right]+c \alpha=0 .
$$

$\left[-p+c+(p+h) \Phi_{i}\left(z_{i}\right)\right]$ should be negative in order for (7) to hold. Hence we cannot determine whether $P_{1}\left(z_{i}, 0\right)$ is positive or negative when $Q_{-i}=0$, that is, $R C_{i}\left(Q_{-i}\right)$ may be increasing or decreasing, which is dependent on the parameters related to demand, capacity, and cost.

\section{References}

Arunachalam, R., \& Sadeh, N. M. (2005). The supply chain trading agent competition. Electronic Commerce Research and Applications, 4, 66-84.

Aviv, Y., \& Federgruen, A. (1998). The operational benefits of information sharing and vendor managed inventory programs. Working Paper, Olin School of Business, Washington University.

Bakal, I. S. (2003). Value of supplier's capacity information in a two-echelon supply chain. MS Thesis, Department of Industrial Engineering, Middle East Technical University.

Basar, T., \& Oldser, G. J. (1995). Dynamic noncooperative game theory (2nd edn.). London/New York: Academic Press.

Cachon, P. G. (2003). Supply chain coordination with contracts. In S. Graves \& T. de Kok (Eds.), Handbooks in operations research and management science: supply chain management. Amsterdam: NorthHolland.

Cachon, P. G., \& Fisher, M. (2000). Supply chain inventory management and the value of shared information. Management Science, 46(8), 1032-1048.

Cachon, P. G., \& Lariviere, M. A. (1999a). Capacity choice and allocation: strategic behavior and supply chain performance. Management Science, 45(8), 1091-1108.

Cachon, P. G., \& Lariviere, M. A. (1999b). An equilibrium analysis of linear, proportional and uniform allocation of scarce capacity. IIE Transactions, 31, 835-849.

Cachon, P. G., \& Lariviere, M. A. (1999c). Capacity allocation using past sales: when to turn-and-earn. Management Science, 45(5), 685-703.

Cachon, P. G., \& Lariviere, M. A. (2001). Contracting to assure supply: how to share demand forecasts in a supply chain. Management Science, 47(5), 629-646.

Cachon, P. G., \& Zhang, F. (2006). Procuring fast delivery: sole sourcing with information asymmetry. Management Science, 52(6), 881-896.

Chen, F. (2003). Information sharing and supply chain coordination. In S. Graves \& T. de Kok (Eds.), Handbooks in operations research and management science: supply chain management. Amsterdam: NorthHolland.

Corbett, C. J. (2001). Stochastic inventory systems in a supply chain with asymmetric information: cycle stocks, safety stocks, and consignment stock. Operations Research, 49(4), 487-500.

Corbett, C. J., \& de Groote, X. (2000). A supplier's optimal quantity discount policy under asymmetric information. Management Science, 46(3), 444-450. 
Corbett, C. J., Zhou, D., \& Tang, C. S. (2004). Designing supply contracts: contract type and information asymmetry. Management Science, 50(4), 550-559.

Cvsa, V., \& Gilbert, S. M. (2002). Strategic commitment versus postponement in a two-tier supply chain. European Journal of Operational Research, 141, 526-543.

Dai, Y., Chao, X., Fang, S. C., \& Nuttle, H. L. W. (2005). Game theoretic analysis of a distribution system with customer market search. Annals of Operation Research, 135, 223-238.

Deshpande, V., \& Schwarz, L. B. (2002). Optimal capacity choice and allocation in decentralized supply chains. Working Paper, Krannert School of Management, Purdue University, W. Lafayette, IN 47907.

Ehrhant, K. M. (2002). A well-known rationing game. Working Paper, Mannheim University.

Gavirneni, S. (2002). Information flows in capacitated supply chains with fixed ordering costs. Management Science, 48(5), 644-651.

Gavirneni, S., Kapuscinski, R., \& Tayur, S. (1999). Value of information in capacitated supply chains. Management Science, 45(1), 16-24.

Gurnani, H., \& Shi, M. (2006). A bargaining model for a first-time interaction under asymmetric beliefs of supply reliability. Management Science, 52(6), 865-880.

Ha, A. Y. (2001). Supplier-buyer contracting: asymmetric cost information and cutoff level policy for buyer participation. Naval Research Logistics, 48, 41-64.

Jemai, Z., Dallery, Y., \& Erkip, N. (2010). Contracting under uncertain capacity: a generalization. International Journal of Inventory Research, 1(2), 125-149.

Karaesmen, F., Liberopoulos, G., \& Dallery, Y. (2004). The value of advance demand information in production/inventory systems. Annals of Operation Research, 126, 135-157.

Kiyotaki, N., \& Moore, J. (1997). Credit chains. Mimeo, London School of Economics.

Lau, A. H., \& Lau, H. (2001). Some two-echelon style-goods inventory models with asymmetric market information. European Journal of Operational Research, 134, 29-42.

Lau, A. H., \& Lau, H. (2005). Some two-echelon supply-chain games: improving from deterministicsymmetric-information to stochastic-asymmetric-information models. European Journal of Operational Research, 161, 203-223.

Lee, H., \& Whang, S. (2000). Information sharing in a supply chain. International Journal of Technology Management, 20, 373-387.

Lee, H., Padmanabhan, V., \& Whang, S. (1997). Information distortion in a supply chain: the bullwhip effect. Management Science, 43(4), 546-558.

Lee, H., So, K., \& Tang, C. (2000). The value of information sharing in a two-level supply chain. Management Science, 46(1), 626-643.

Li, H., Ritchken, P., \& Wang, Y. (2009). Option and forward contracting with asymmetric information: valuation issues in supply chains. European Journal of Operational Research, 197, 134-148.

Lim, W. (2001). Producer-supplier contracts with incomplete information. Management Science, 47(5), 709_ 715.

Lippman, S. A., \& McCardle, K. F. (1997). The competitive newsboy. Operations Research, 45(1), 54-65.

Liu, X., \& Çetinkaya, S. (2009). Designing supply contracts in supplier vs buyer-driven channels: the impact of leadership, contract flexibility and information asymmetry. IIE Transactions, 41, 687-701.

Mallik, S., \& Harker, P. (2004). Coordinating supply chains with competition: capacity allocation in semiconductor manufacturing. European Journal of Operational Research, 159, 330-347.

Rong, Y., Shen, Z.-J. M., \& Snyder, L. V. (2008). Bullwhip and reverse bullwhip effects under the rationing game. Working Paper, P.C. Rossin College of Engineering and Applied Sciences, Lehigh University, Bethlehem, PA.

Schneeweiss, C., \& Zimmer, K. (2004). Hierarchical coordination mechanisms within the supply chain. European Journal of Operational Research, 153, 687-703.

Wang, Y., \& Gerchak, Y. (2001). Supply chain coordination when demand is shelf-space dependent. Manufacturing \& Service Operations Management, 3(1), 82-87.

Wang, J., Lau, H., \& Lau, A. H. (2008). How a retailer should manipulate a dominant manufacturer's perception of market and cost parameters. International Journal of Production Economics, 116, 43-60.

Wang, J., Lau, H., \& Lau, A. H. (2009). When should a manufacturer share thruthful manufacturing cost information with a dominant retailer? European Journal of Operational Research, 197, 266-286.

Yang, Z., Aydın, G., Babich, V., \& Beil, D. R. (2009). Supply disruptions, asymmetric information, and a backup production option. Management Science, 55(2), 192-209. 